\title{
Mobile Robot Navigation and Obstacle-avoidance using ANFIS in Unknown Environment
}

\author{
Mohammed Algabri \\ Dept. of Computer Science \\ College of computer and information \\ sciences \\ King Saud University, Riyadh, Saudi \\ Arabia
}

\author{
Hassan Mathkour \\ Dept. of Computer Science \\ College of computer and information \\ sciences \\ King Saud University, Riyadh, Saudi \\ Arabia
}

\author{
Hedjar Ramdane \\ Dept. of Computer Engineering \\ College of computer and information \\ sciences \\ King Saud University, Riyadh, Saudi \\ Arabia
}

\begin{abstract}
Navigation and obstacle avoidance in an unknown environment is proposed in this paper using hybrid neural network with fuzzy logic controller. The overall system is termed as Adaptive Neuro Fuzzy Inference System (ANFIS). ANFIS combines the benefits of fuzzy logic and neural networks for the purpose of achieving robotic navigation task. Simulation results are presented using Khepera Simulator (KiKs) within MATLAB environment. Moreover, experimental results are obtained using Khepera III platform.
\end{abstract}

\section{Keywords}

Fuzzy logic controller, neural network, ANFIS, mobile robot navigation Khepera III.

\section{INTRODUCTION}

Several successful navigation approaches based on fuzzy neural network algorithms have been suggested in the literature. In [1], authors use genetic algorithm learning to optimize the navigation of Khepera mobile robot in a dynamic environment. Computer simulation is carried by (KIKS arena) with a number of obstacles. The measure of success used in this work is called Error Penalty (EP), the results show an $\mathrm{EP}=12.58$ for fuzzy logic controller and $\mathrm{EP}=9.73$ for the proposed controller. It was shown in the article that the output of the proposed adapted controller is superior to a simplistic fuzzy controller. Another novel approach for mobile robot control is proposed in [2], this approach uses a neural network to choose the optimum number of rules to reduce the computation time in real time application. CPU time consumed in the execution of the control loop is used as a measure in this work. Experimental results show increased response of controller (as compared to previously proposed fuzzy logic controllers) and improved performance in terms of collision avoidance. In [3], a dynamic neuro-fuzzy system with short memory for obstacle avoidance in unknown environment is presented. Simulation environment with size of Length (45) $\mathrm{m}$ and (40) $\mathrm{m}$ for width, the Length of robot is (1.5) $\mathrm{m}$ and the width (1) m. Results show that this proposed method can make the robot reach the destination point via an optimal path while successfully avoiding obstacles for a particular environment as compared to a conventional fuzzy-rule based system. In [4], authors developed a fuzzy-neural network based for building a system that operates in dynamic environment. Back Propagation Artificial Neural Network can be trained via patterns that are generated by simulation thus saving precious training time. Another neuro-fuzzy approach was proposed in [5] for real time mobile robot navigation. A neural network learning algorithm is used to tune the parameters of membership functions. This turns the trajectory generated by the fuzzy-logic system much smoother. The simulation, using a Robotsim version 1.0.03, shows that this method reduces number of rules to 48 fuzzy rules, much fewer than the approaches that employ hundreds of rules.

In our work, ANFIS is applied as a controller for the mobile robot navigation and obstacle avoidance in an unknown environment. Moreover, ANFIS controller is compared with a conventional fuzzy logic controller to present the effectiveness of our proposed system. This paper is divided into four sections. Section 1 presents the introduction and literature review. Section 2 presents an ANFIS controller. Section 3, 4 present simulation and experimental results and Section 5 shows the conclusion.

\section{ANFIS CONTROLLER}

As we know, fuzzy systems present a host of problems in terms of modeling and implementing membership function and rules. ANFIS is our attempt to address a range of such issues. ANFIS is an integrated system of Takagi-Sugeno fuzzy inference system (FIS) and artificial neural network (ANN) [6]. The ANFIS learns the rules and tuning the membership functions from the dataset. Our proposed method uses a multilayer ANFIS controller. Two of these layers are dedicated to motion behavior and two of these serve to render obstacle avoidance behavior. This architecture is shown in figure (1). 


\section{Motion behavior}

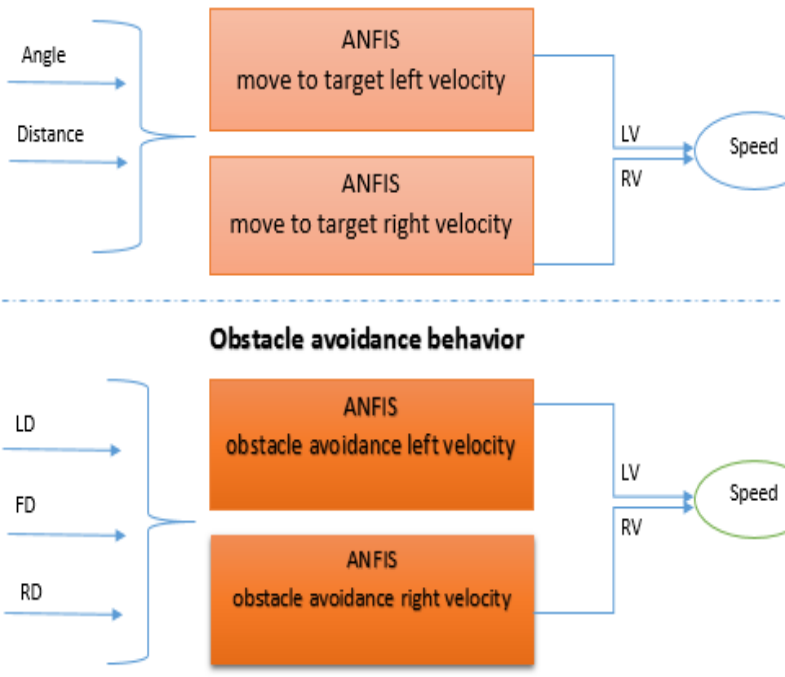

Fig. 1: General structure of the proposed method.

As stated earlier, figure (1) presents the general architecture of a hybrid neuro- fuzzy system. Our system exhibits two behaviors (i) motion to the target (MTT behavior) and (ii) obstacle avoidance. Since Sugeno model has only one output, four separate Sugeno models are designed. Here two of these models are used to control left and right wheel velocities during MTT behavior and the remaining two are used for control of left and right wheel velocities during obstacle avoidance behavior. Heading angle between robot and target (Angle) and distance between robot and target (Distance) are the inputs for MTT behavior. Left distance to obstacle (LD), front distance (FD), and right distance (RD) are the inputs for obstacle avoidance behavior.

\subsection{ANFIS for Motion to the Target Behavior}

Table (1) shows a sample of training, checking and testing dataset that is used in the learning process employed by ANFIS for the MTT behavior.

We used five Gaussian membership functions and a hybird learning method with 5000 Epochs. In the hybrid learning, the learning takes place in two directions on a nural network forward (from inputs towards outputs) and backward (from outputs towards inputs). In the forward pass the algorithm uses least-squares method to identify the consequent parameters on the last layer. In the backward pass the errors are propagated backwards and the premise parameters are updated by gradient descent. The tuned membership functions are shown in figures $(4,5)$
Table 1: Samples of training, checking, and testing data.

\begin{tabular}{|r|r|r|r|}
\hline $\begin{array}{c}\text { Angle (heading } \\
\text { angle between } \\
\text { robot and } \\
\text { target) }\end{array}$ & $\begin{array}{c}\text { Distance } \\
\text { (distance } \\
\text { between robot } \\
\text { and target) }\end{array}$ & $\begin{array}{c}\text { LV (Left } \\
\text { Wheel } \\
\text { Velocity) }\end{array}$ & $\begin{array}{c}\text { RV (Right } \\
\text { Wheel } \\
\text { Velocity) }\end{array}$ \\
\hline-5.54566 & 125.6717 & 9.089895 & 9.216776 \\
\hline-150.381 & 100.1263 & 3.11724 & 10.3825 \\
\hline 48.91731 & 89.4665 & 9.950463 & 5.000517 \\
\hline-40.3541 & 87.27249 & 5.001848 & 7.999829 \\
\hline-120.131 & 112.3009 & 3.014434 & 9.798993 \\
\hline 78.43583 & 84.18441 & 9.739525 & 3.086601 \\
\hline 164.3489 & 85.29204 & 10.95403 & 3.007601 \\
\hline 180 & 33.01147 & 10.76709 & 3.00633 \\
\hline-180 & 33.75196 & 3.005514 & 10.90095 \\
\hline 2.898077 & 37.94814 & 4.997442 & 4.99673 \\
\hline & & & \\
\hline
\end{tabular}

Figure (2) shows the training results, the sample of 49 pairs are used of training of the left velocity for MTT behavior. The error rate 0.585 is on the 5000 epochs.

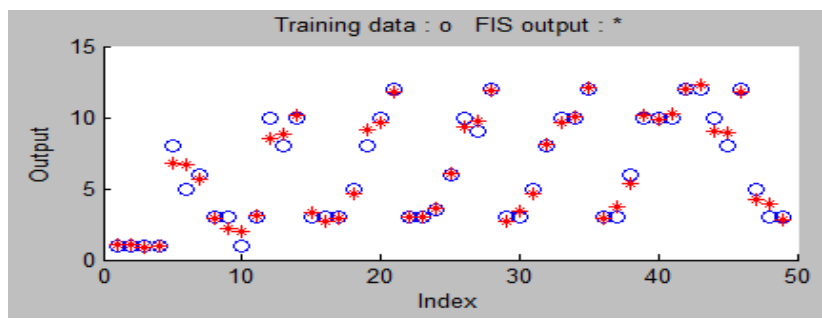

Fig. 2: training error of the MTT behavior.

Figure (3) shows the testing results, the sample of 25 pairs are used of testing of the left velocity for MTT behavior. The error rate 0.766 is on the 5000 epochs.

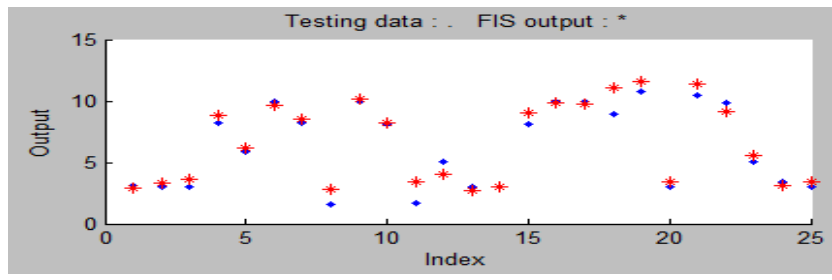

Fig. 3: testing error of the MTT behavior.
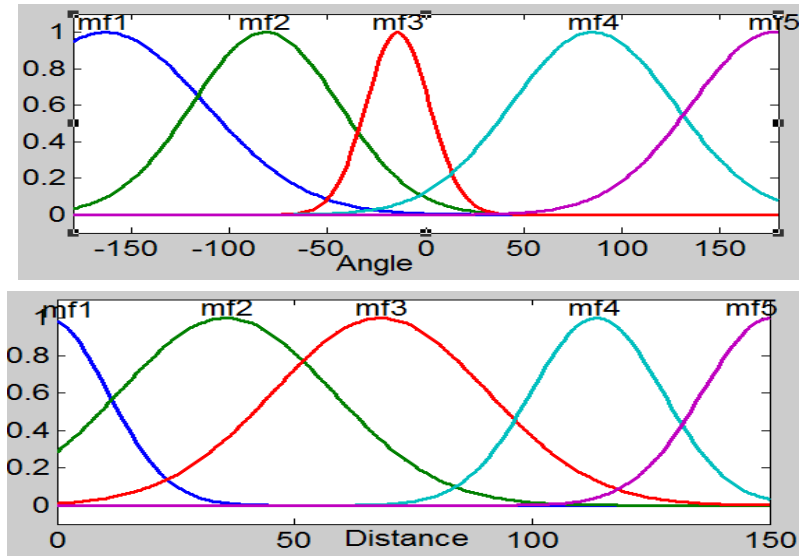

Fig. 4: Membership function of left velocity after the learning step for MTT behavior 

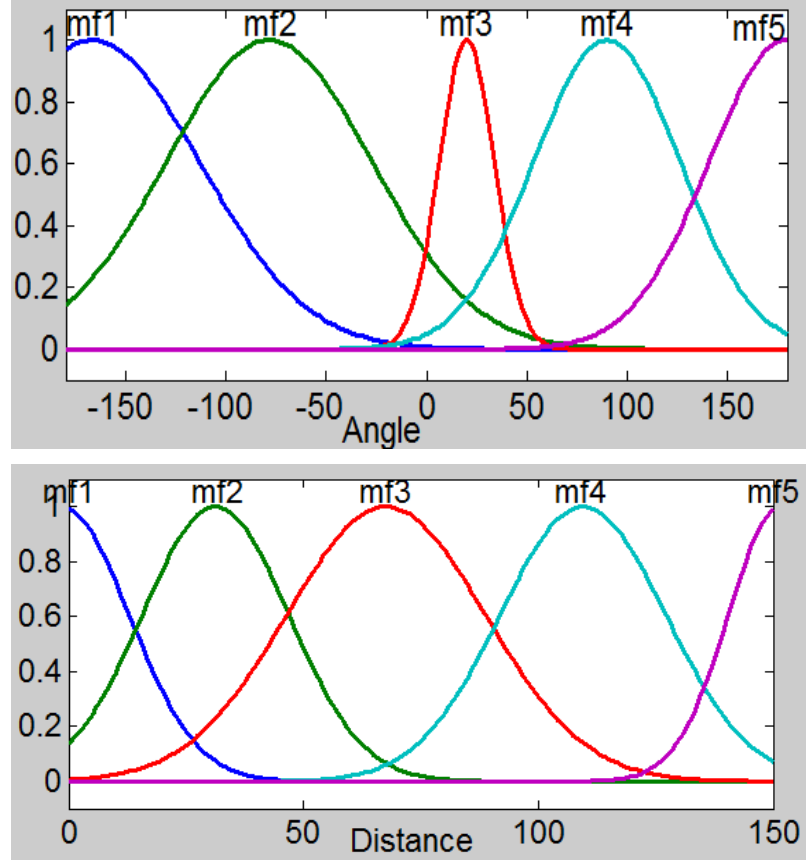

Fig. 5: Membership function of right velocity after the learning step for MTT behavior.

\subsection{ANFIS for Obstacle-avoidance Behavior}

Table (2) depicts a sample of training, checking and testing dataset that is used in the learning process employed by ANFIS

Table 2 : Samples of training, checking, and testing data. for the Obstacle-avoidance behavior.

\begin{tabular}{|r|r|r|r|r|}
\hline $\begin{array}{c}\text { LD (Left } \\
\text { Distance) }\end{array}$ & $\begin{array}{c}\text { FD (Front } \\
\text { Distance) }\end{array}$ & $\begin{array}{c}\text { RD (Right } \\
\text { Distance) }\end{array}$ & $\begin{array}{c}\text { LV (Left } \\
\text { Velocity) }\end{array}$ & $\begin{array}{c}\text { RV (Right } \\
\text { Velocity) }\end{array}$ \\
\hline 2 & 80 & 5 & -4 & -4 \\
\hline 5 & 5 & 70 & 1 & 4.5 \\
\hline 50 & 5 & 4 & -4 & -3 \\
\hline 35 & 40 & 80 & 1.5 & 3.5 \\
\hline 70 & 4 & 3 & -2 & -3 \\
\hline 80 & 40 & 45 & 5 & 2 \\
\hline 70 & 80 & 1 & 3 & 1 \\
\hline 70 & 70 & 70 & 1 & 1 \\
\hline 69 & 2 & 78 & -2 & -2 \\
\hline 9 & 70 & 45 & 0 & 4 \\
\hline & & & & \\
\hline
\end{tabular}

Figure (6) shows the training results, the sample of 27 pairs are used of training of the right velocity for Obstacle-avoidance behavior. The error rate 0.0000358 is on the 5000 epochs.
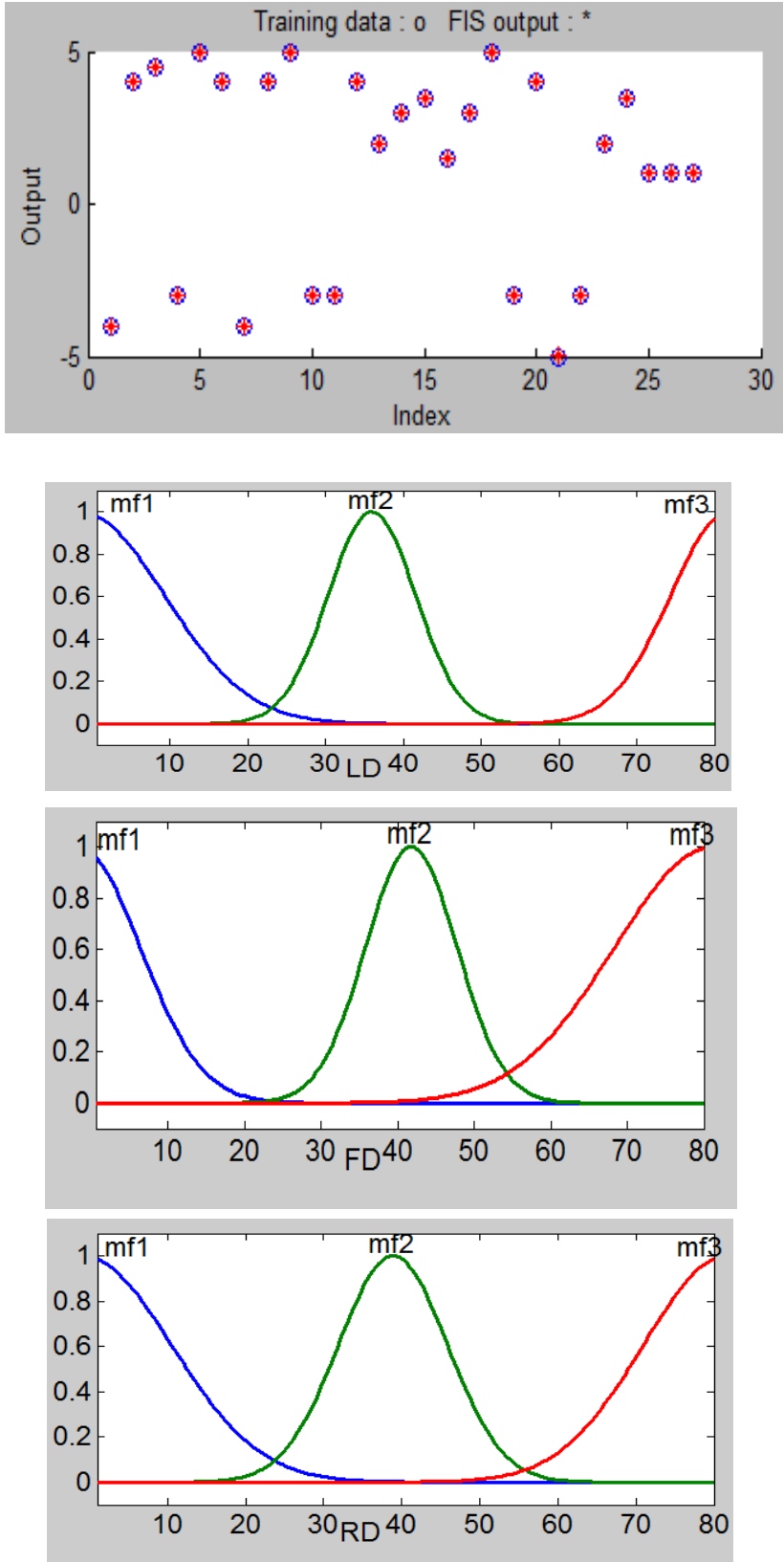

Fig. 6: Training error of Obstacle-avoidance behavior.

Figure (7) shows the testing results, the sample of 27 pairs are used of testing of the right velocity for Obstacle-avoidance behavior. The error rate is 1.469 on the 5000 epochs.

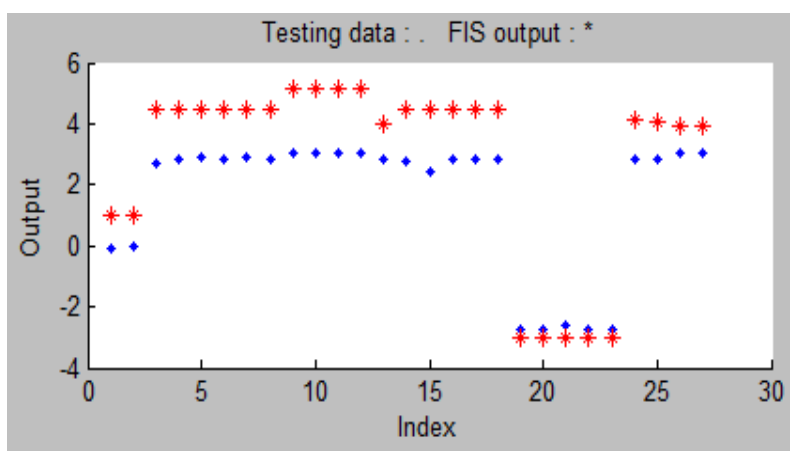

Fig. 7: Testing error of Obstacle-avoidance behavior. 
We used three Gaussian membership functions and a hybird learning method with 5000 Epochs. The tuned membership functions are shown in figures $(8,9)$.
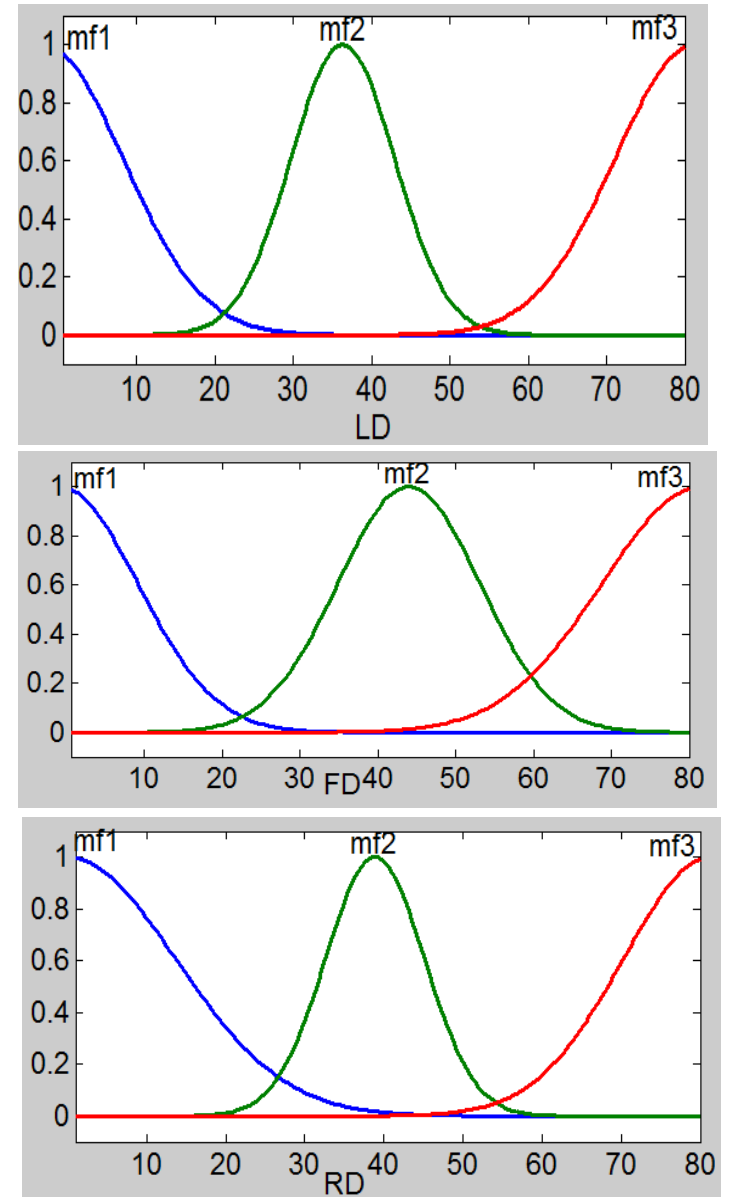

Fig. 8: Membership function of left velocity after the learning step for Obstacle avoidance behavior
Volume 91 - No.14, April 2014
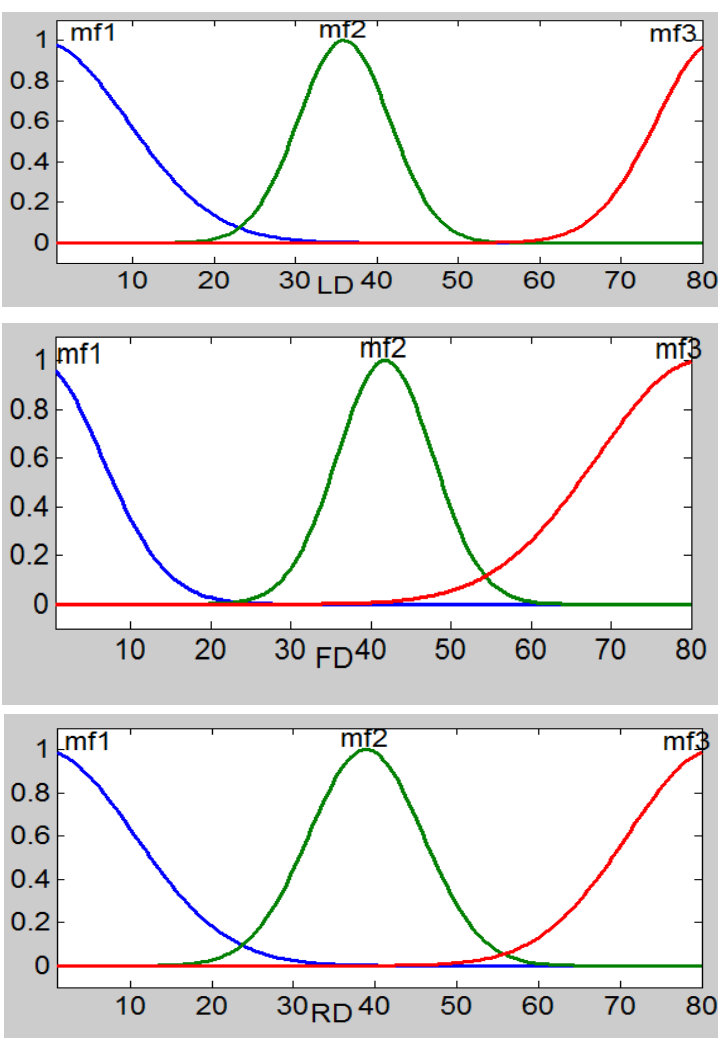

Fig. 9: Membership function of right velocity after the learning step for Obstacle avoidance behavior.

\section{SIMULATION RESULT}

The simulation results are obtained by using Khepera simulator KiKs [7]. KIKS is a matlab application that simulates a Khepera robot to imitate its true physical motion. Figure (10) shows the robot navigation in the environment without obstacles. Figures $(11,12)$ illustrate the robot navigation and obstacle avoidance in an unknown environment.

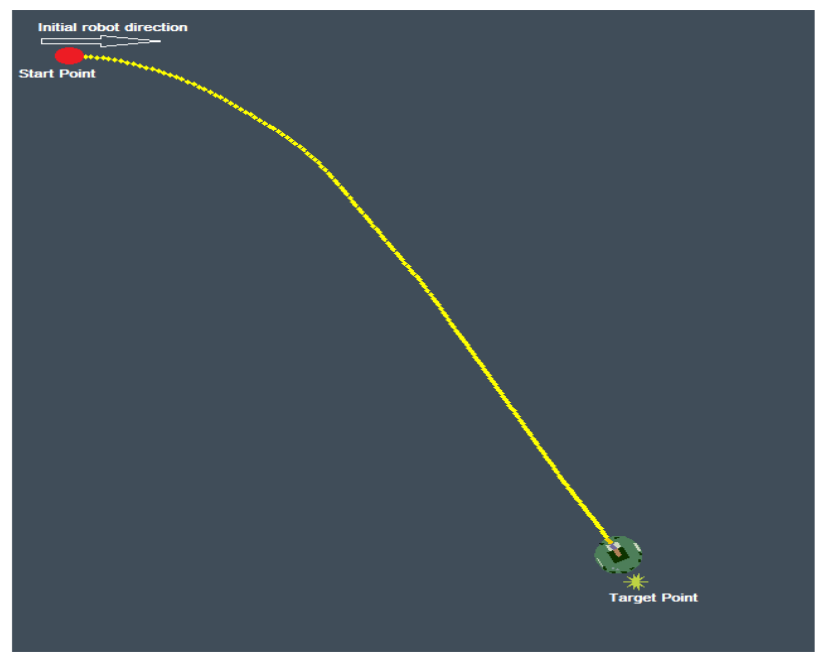

Figure 10: Robot navigation in an environment without obstacles. 


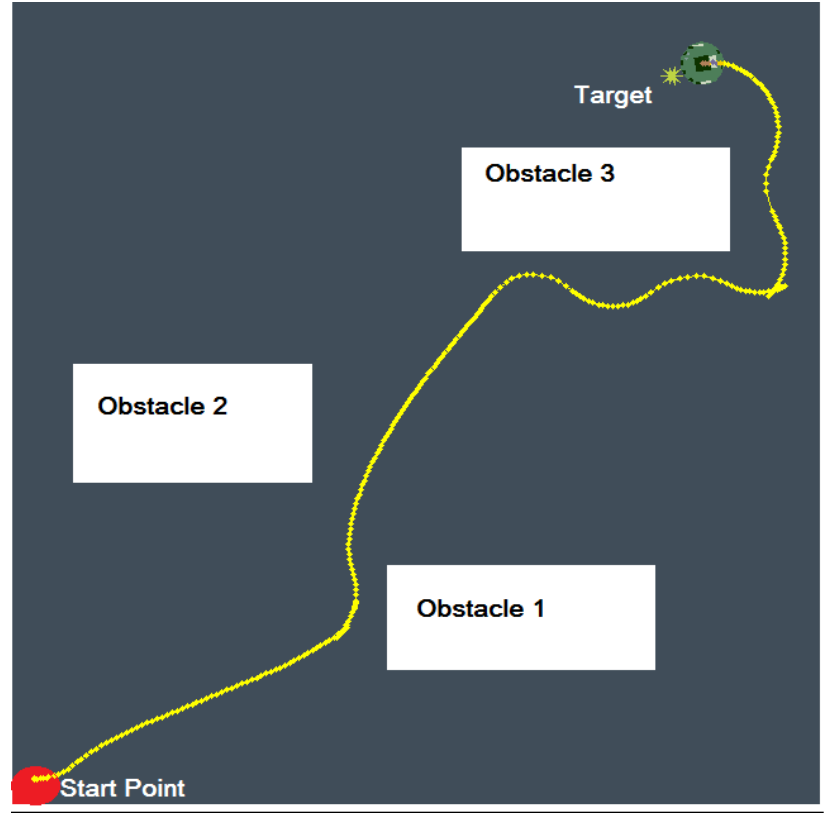

Fig. 11: robot navigation in an environment with scattered obstacles.

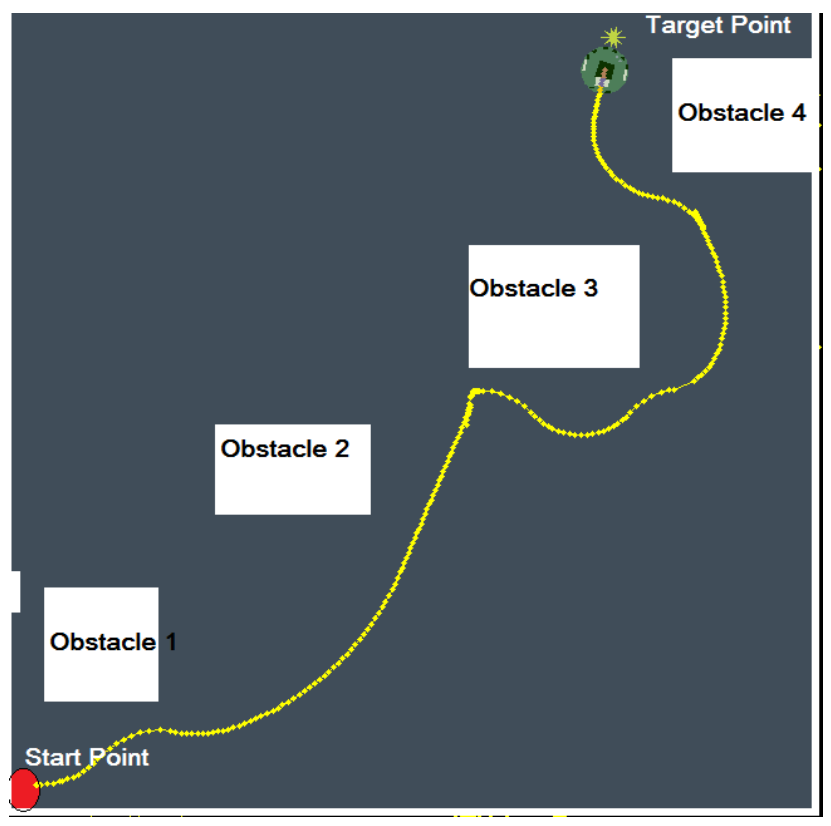

Fig. 12: robot navigation in an environment with four obstacles.

By observing the followed trajectory, it is evident that the path followed by the robot is sub-optimal. Nevertheless it can be contested that this algorithm mitigates the typical difficulties involved in design and implementation of fuzzy logic membership functions. The execution speed of the control loop remains within the real-time execution bounds i.e. $<100$ milliseconds.

\section{EXPERIMENTAL RESULT}

In this paper, we used Khepera III platform to obtain the proposed method in real environment. Figure 13 illustrates the Khepera III platform. The robot is fully controlled from a remote Matlab program running on a PC.

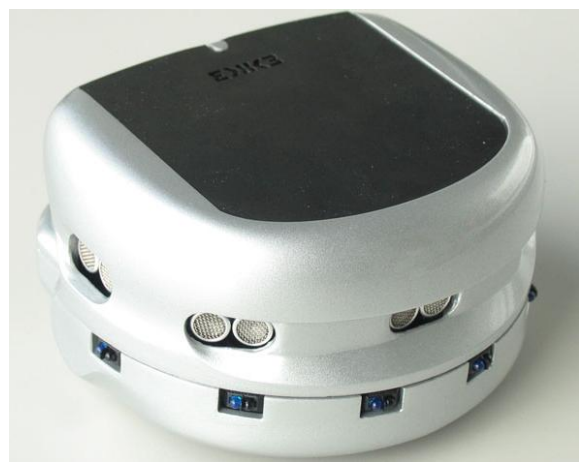

Fig. 13: Khepera III.

The first scenario show in figures 14-17, the method examined to navigate the robot in environment without obstacles. The robot should be move from start point $(0,0)$ to target point $(0$, $1000)$, i.e. one meter on y-axis.

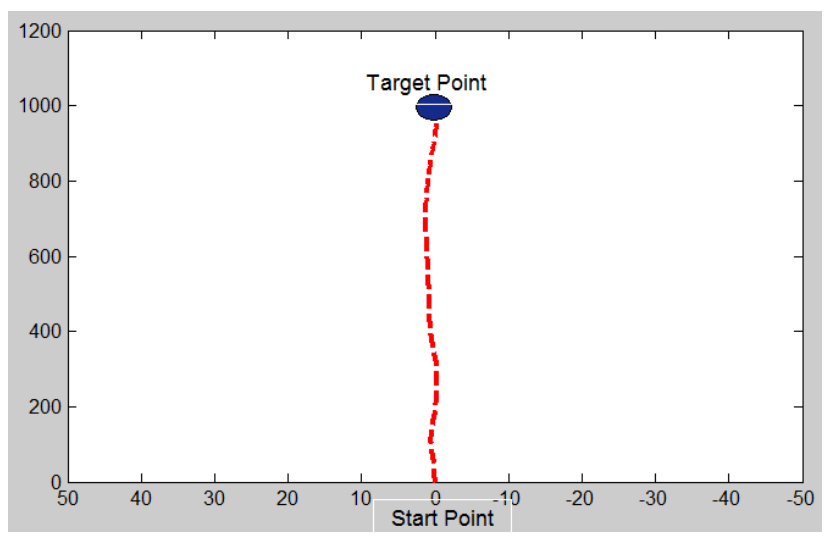

Fig. 14: Robot trajectory in environment without obstacle.

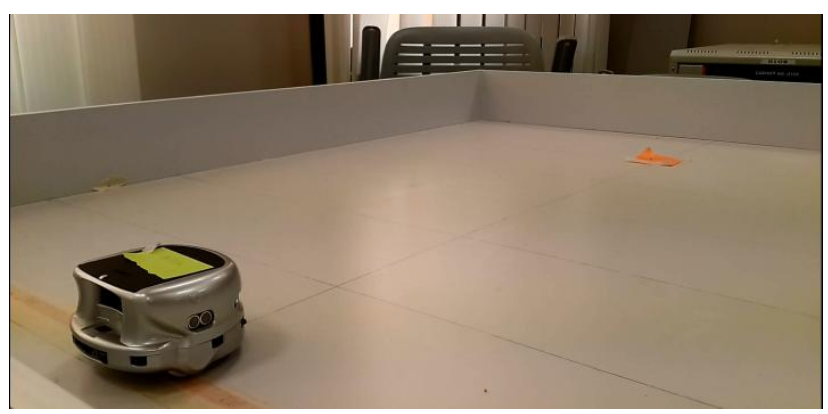

Fig. 15: Robot in start point.

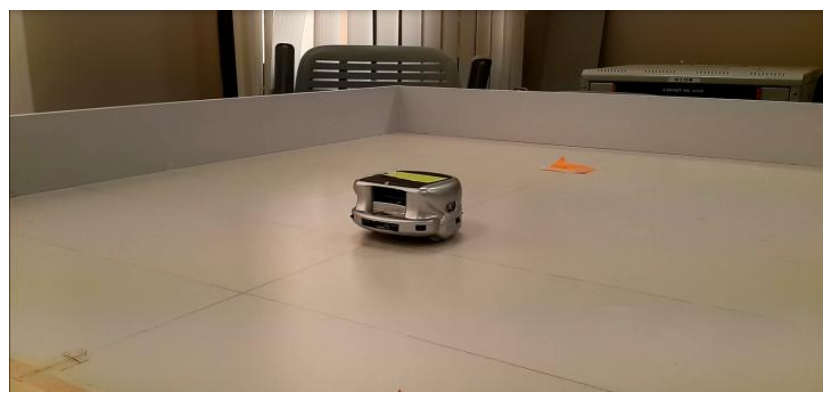

Fig. 16 : Robot during navigation to target. 


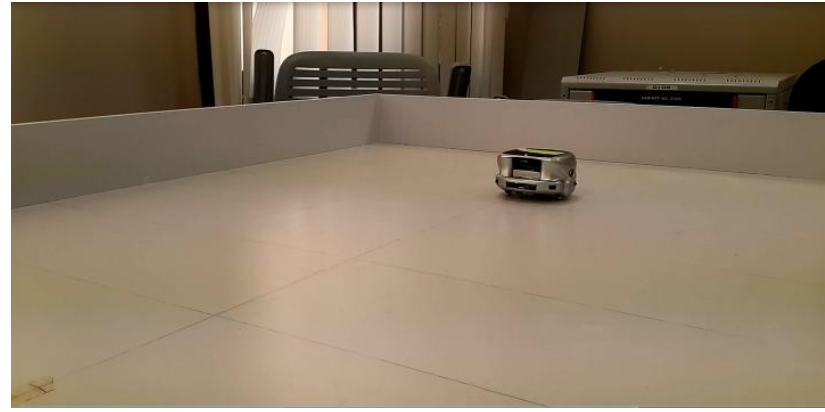

Fig. 17 : Robot reach target.

In the second scenario, we examined the method to navigate robot in unknown environment without obstacles. Figures 1821 show the trajectory of robot in this scenario and the robot while avoiding the obstacle.

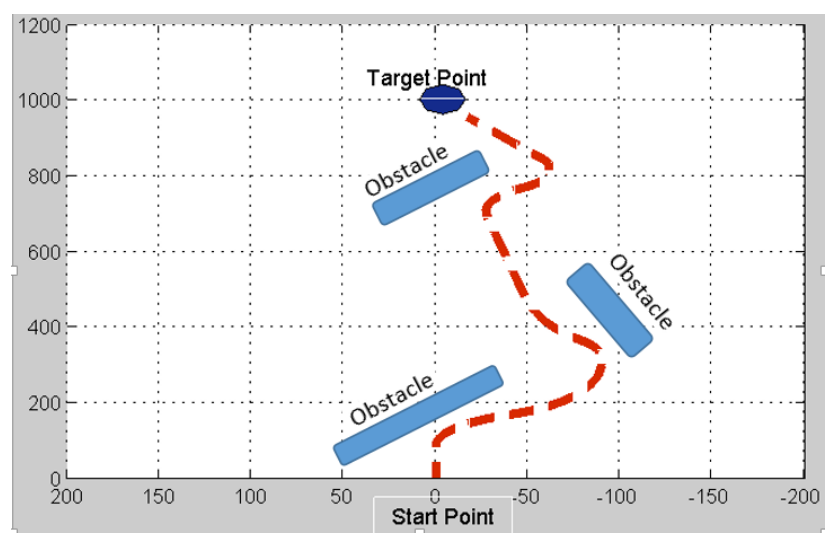

Fig. 18: Robot trajectory in environment with obstacles.

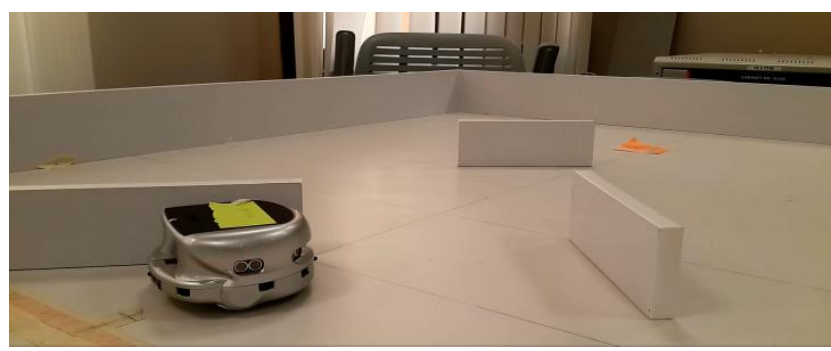

Fig. 19 : Robot during avoiding first obstacle.

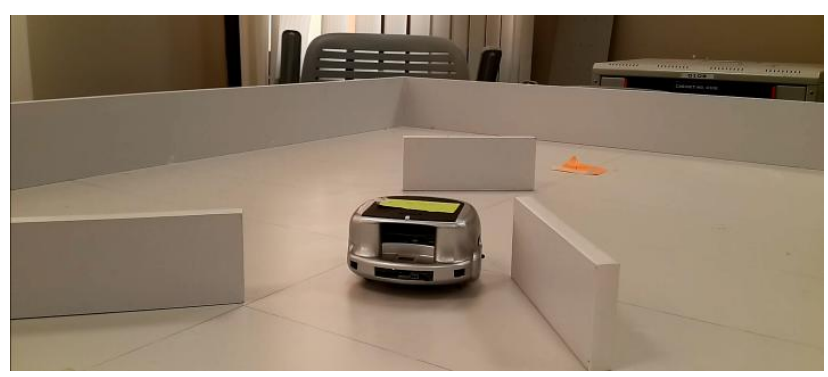

Figure 20 : Robot during avoiding second obstacle.

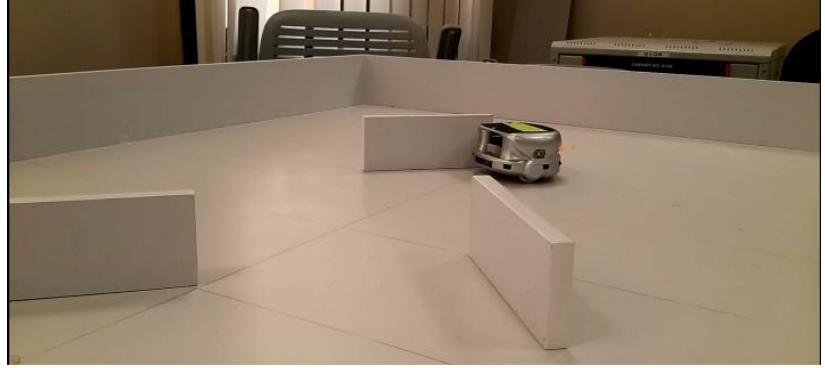

Figure 21 : Robot during avoiding third obstacle.

\section{CONCLUSION}

Navigation of mobile robot and obstacles avoidance has been discussed in this paper using ANFIS system. Experimental and Simulation results show the effectiveness and the robustness of the proposed method in an unknown environment. The parameter adjustment of membership functions after application of a hybrid learning technique has proven its effectiveness as replacement of manual parameter tuning of membership functions. The work can be further improved by application and experimentation with superior supervised/un-supervised learning techniques within the ANFIS architecture.

\section{ACKNOWLEDGMENTS}

This work is supported by NPST program by King Saud University (Project No. : 08-ELE-300-02).

\section{REFERENCES}

[1] Obe, O. and Dumitrache, I. 2012. "Adaptive Neuro-Fuzzy Controler With Genetic Training For Mobile Robot Control," International Journal of Computers Communications \& Control, vol. 7, no. 1, pp. 135-146.

[2] AbuBaker, A. 2012. "A Novel Mobile Robot Navigation System Using Neuro-Fuzzy Rule-Based Optimization Technique," Research Journal of Applied Sciences, vol. 4.

[3] Chen, C. and Richardson, P.2012. "Mobile robot obstacle avoidance using short memory: a dynamic recurrent neurofuzzy approach," Transactions of the Institute of Measurement and Control, vol. 34, no. 2-3, pp. 148-164.

[4] Kundu, S., Parhi, R., Deepak ,B.B.V.L. 2012. "FuzzyNeuro based Navigational Strategy for Mobile Robot," International Journal of Scientific \& Engineering Research, Volume 3, Issue 6.

[5] Zhu, A. and Yang, S. X. 2007. "Neurofuzzy-based approach to mobile robot navigation in unknown environments," Systems, Man, and Cybernetics, Part C: Applications and Reviews, IEEE Transactions on, vol. 37, no. 4 , pp. $610-621$.

[6] Jang, J.S.R. 1993. ANFIS: Adaptive-network-based fuzzy inference systems.,IEEE Trans. on Syst. Man, and Cybern., vol.23 no.5, pp. 665-685.

[7] KiKS is a Khepera Simulator. http://www.theodorstorm.se/index/2866.html (02, June, 2013, date accessed) 